\title{
Dopamine/Silica Nanoparticle Assembled, Microscale Porous Structure for Versatile Superamphiphobic Coating
}

Fang LI, Miao DU*, Qiang ZHENG

MOE Key Laboratory of Macromolecular Synthesis and Functionalization, Department of Polymer Science and Engineering, Zhejiang University, Hangzhou, 310027, China

Figure S1. Photographs of polydopamine bound HiFSNs cluster dispersions and HiFSNs dispersion.

Figure S2. Generally accepted polymerization of dopamine and further chemical modification to polydopamine.

Figure S3. TEM, EDX and DLS data for HiFSNs and polydopamine bound HiFSNs clusters.

Figure S4. FT-IR spectra of polydopamine bound HiFSNs clusters.

Figure S5. PCM images taken during the cooling processing to the polydopamine bound HiFSNs cluster dispersion.

Figure S6. Photographs of coatings after freeze-drying process.

Figure S7. FT-IR spectra of the polydopamine bound HiFSNs clusters after being treated with two-step CVD.

Figure S8. SCAs for microscale porous coatings treated with one-step CVD and twostep CVD and impinged by water.

Figure S9. High resolution XPS spectra for C 1s of the microscale porous coatings after CVD.

Figure S10. Photograph of a water droplet deposited on the quasi-superamphiphobic coating.

Figure S11. Schematic illustration for a droplet deposited on the porous structure.

Figure S12. Schematic illustration for calculating the air fraction ( $\left.\phi_{\text {air }}\right)$. 
Figure S13. Microscale porous superamphiphobic coatings on different materials.

Table S1. Weight loss for polydopamine bound HiFSNs clusters with different initial dopamine concentrations. 
By increasing the initial dopamine concentration, the dispersion color turned from light blue (due to the Rayleigh scattering caused by HiFSNs) to brown or black, which was the characteristic color of polydopamine (Figure S1A). The bound HiFSNs clusters were all well dispersed and the obtained dispersions were stable and uniform (Figure S1B and C). The concentration of initial HiFSNs was 1:100, wt/wt.

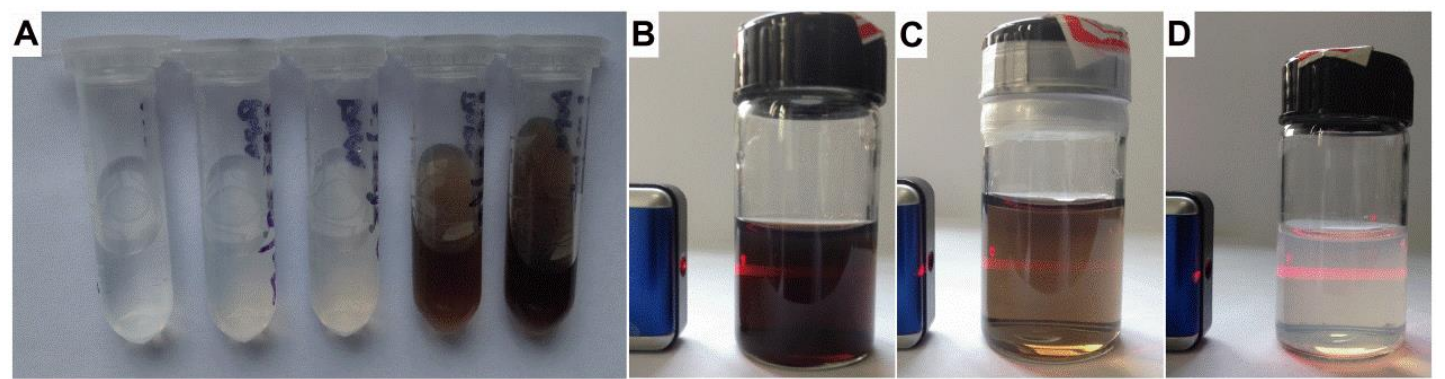

Figure S1. Photographs of polydopamine bound HiFSNs cluster dispersions and HiFSNs dispersion. (A) Photograph of polydopamine bound HiFSNs cluster dispersions with different primary dopamine concentrations (Left to right, 0, 0.1, 0.5, 1.0, $2.0 \mathrm{mg} / \mathrm{mL}$ ). Polydopamine bound HiFSNs cluster dispersions with (B) $2.0 \mathrm{mg} / \mathrm{mL}$, (C) $1.0 \mathrm{mg} / \mathrm{mL}$, and (D) $0 \mathrm{mg} / \mathrm{mL}$ primary dopamine concentration. All dispersions showed Tyndall effect. 


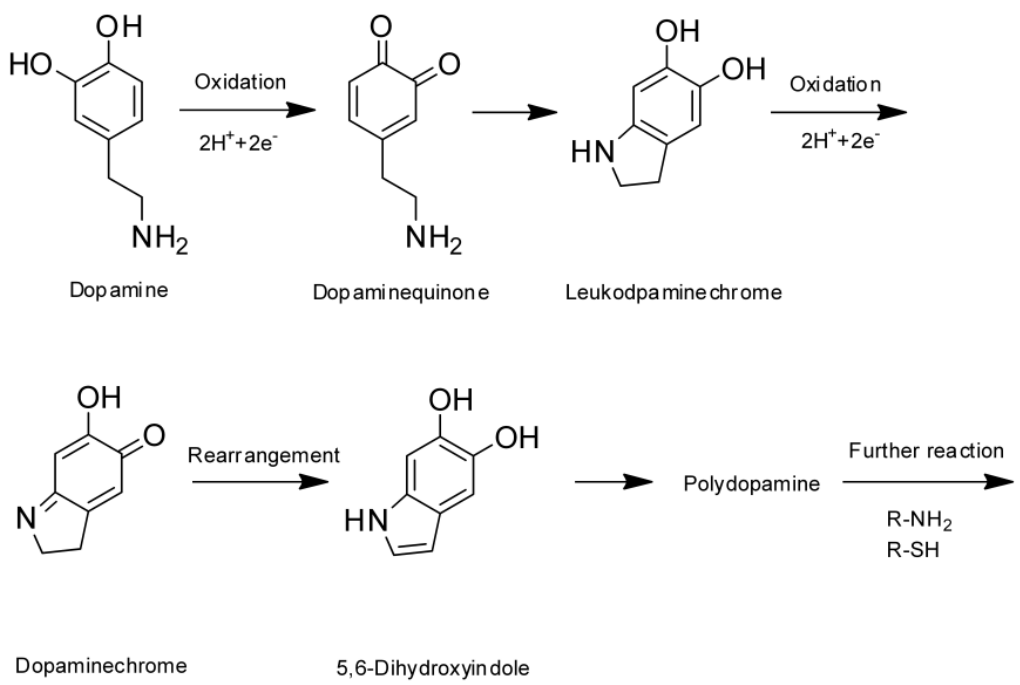

Figure S2. Generally accepted polymerization of dopamine and further chemical modification to polydopamine. ${ }^{1}$ 

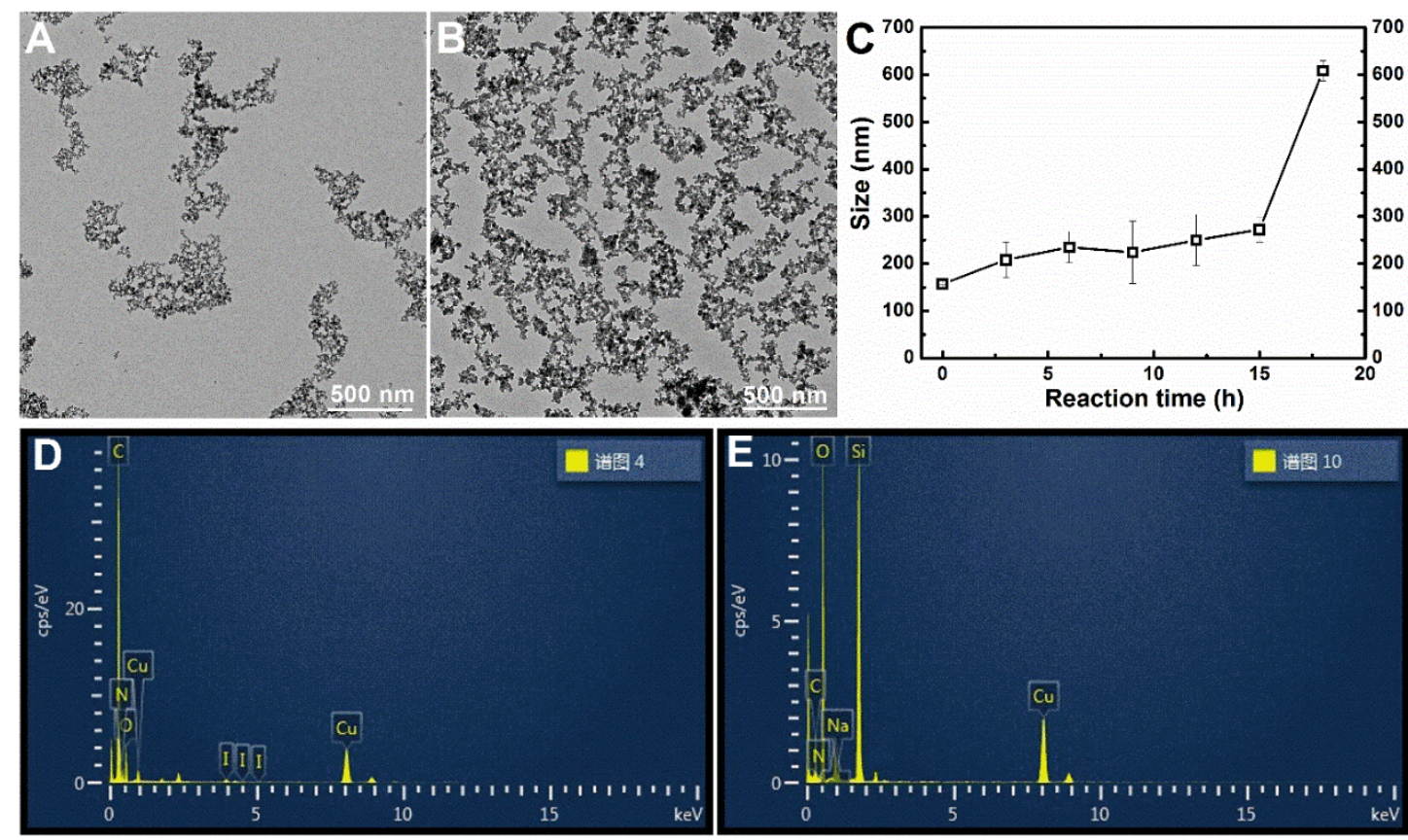

Figure S3. TEM, EDX and DLS data for HiFSNs and polydopamine bound HiFSNs clusters. TEM images of (A) HiFSNs aggregates and (B) the polydopamine bound HiFSNs cluster aggregates. (C) Size of the polydopamine bound HiFSNs clusters during the reaction. EDX spectra for (D) polydopamine (Zone I in Figure 2D and E) and (E) polydopamine bound HiFSNs clusters (Zone II in Figure 2D and E). 


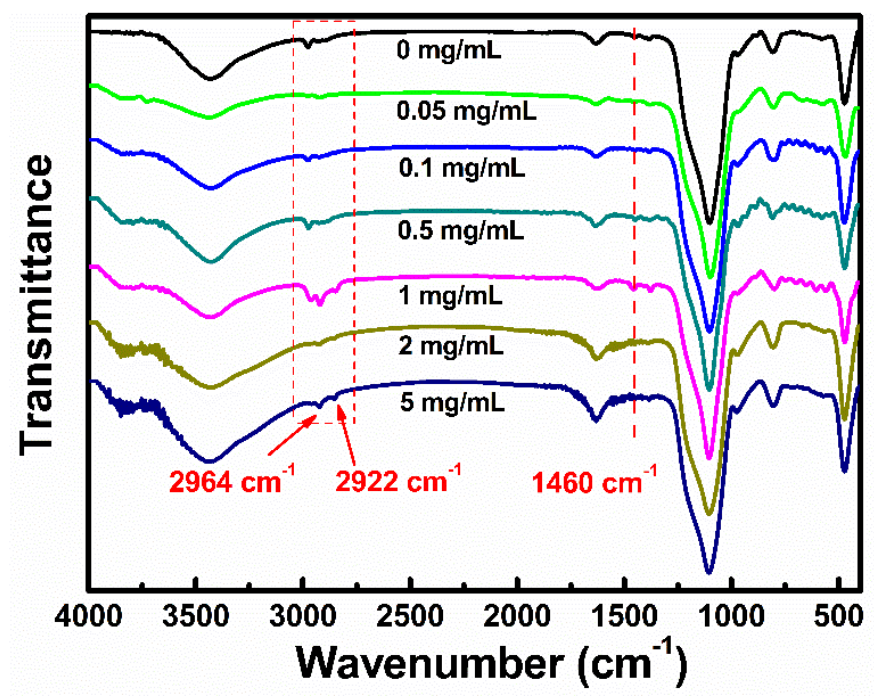

Figure S4. FT-IR spectra of polydopamine bound HiFSNs clusters with different primary dopamine concentrations. The initial HiFSNs concentration is $1: 100, \mathrm{wt} / \mathrm{wt}$.

After Dispersion A $(1.0 \mathrm{mg} / \mathrm{mL}$ of dopamine, 1:100, wt/wt of HiFSNs aqueous dispersion) and Solution $\mathrm{B}\left(100 \mathrm{mM} \mathrm{NaIO}_{4}\right.$ aqueous solution) were mixed and reacted for $18 \mathrm{~h}$. The polydopamine bound HiFSNs clusters were separated from the dispersion via centrifuging. And the polydopamine bound HiFSNs clusters were washed for three times by water to guarantee that the free polydopamine particle and might-unreacted dopamine was completely removed. The particles were dried under $\mathrm{N}_{2}$ flow and then dried in the oven at $50{ }^{\circ} \mathrm{C}$ and were used for TEM and FT-IR analysis.

After carefully observing TEM images of the pure HiFSNs and polydopamine bound HiFSNs clusters, obvious differences could be found out (Figure 2C-E). White arrows in Figure 2D and E clearly point out the polydopamine layer. These regions have been marked in red and denoted as Zone I. Energy dispersive spectrometer (EDX) spectra for polydopamine (Zone I) is manifested in Figure 2B and Figure S3D. $N$ peak clearly indicates the existence of the polydopamine. $N$ atom occupation in the dopamine 
molecule is approx. $9 \mathrm{wt} \%$, being slightly larger than the minimum detection limit of the device (approx. $5 \mathrm{wt} \%$ ). Regions where cannot clearly find out the polydopamine are marked in cyan and denoted as Zone II (Figure 2D and E). EDX spectra for Zone II is displayed in Figure S3E. $N$ peak is even weaker than what is acquired for pure polydopamine layer, but it can still be resolved by the device.

The data for each FT-IR curve has been normalized (Figure S4), taking the characteristic peak at $1105 \mathrm{~cm}^{-1}$ (stretching vibration of $\mathrm{Si}-\mathrm{O}-\mathrm{Si}$ ) as the standard point. The raw HiFSNs bought from Degussa AG showed $\mathrm{CH}_{3}\left(2964 \mathrm{~cm}^{-1}\right)$ and $\mathrm{CH}_{2}(2922$ $\mathrm{cm}^{-1}$ ) stretching vibration peak in the FT-IR spectrum. It means that there are still a few $\mathrm{CH}_{3}$ and $\mathrm{CH}_{2}$ groups on the HiFSNs surfaces. The large $\mathrm{OH}$ stretching vibration peak $\left(3438 \mathrm{~cm}^{-1}\right)$ demonstrates that $\mathrm{OH}$ groups occupy the majority area of the HiFSNs surfaces. Therefore, HiFSNs can be well dispersed in water or aqueous solutions. As compared the FT-IR spectrum of raw HiFSNs with the spectrum of HiFSNs treated with $0.05 \mathrm{mg} / \mathrm{mL}$ primary dopamine, the $\mathrm{OH}, \mathrm{CH}_{3}$, and $\mathrm{CH}_{2}$ stretching vibration peaks are all weakened (Figure S4). With further increasing initial dopamine concentration, three characteristic peaks at $2964 \mathrm{~cm}^{-1}\left(\mathrm{CH}_{3}\right.$ stretching vibration $), 2922 \mathrm{~cm}^{-1}\left(\mathrm{CH}_{2}\right.$ stretching vibration), and around $1460 \mathrm{~cm}^{-1}\left(\mathrm{CH}_{2}\right.$ scissoring vibration and $\mathrm{CH}_{3}$ asymmetric bending vibration) strengthen for the bound HiFSNs clusters. These results reveal that HiFSNs are indeed bound by polydopamine.

Bound polydopamine onto HiFSNs could also be verified by the successively increased weight loss with the increase of the initial dopamine concentration via TGA (Table S1). Both the results indicated that primary HiFSNs are indeed cohered together 
by polydopamine via strong interactions. The enlargement of the bound HiFSNs cluster size in the dispersion can be confirmed by dynamic light scattering (DLS) test (Figure S3C). As solvent evaporation would generate significant aggregation of the HiFSNs or polydopamine bound HiFSNs clusters, TEM images could not reflect the exact size of the particles in the dispersion. DLS data might not reflect the exact particle size in the dispersion, but it still indicates that the size of the polydopamine bound HiFSNs clusters increases with increasing reaction time which demonstrates that HiFSNs are indeed bound by polydopamine. In addition, by increasing the initial dopamine concentration, the dispersion color turns from light blue (due to the Rayleigh scattering caused by HiFSNs) to brown and black, revealing the increase of polydopamine (Figure S1A). The bound HiFSNs clusters with different initial dopamine concentrations are all well dispersed and the obtained dispersions are stable and uniform (Figure S1B and C) on the premise of the initial HiFSNs concentration of 1:100, wt/wt.

Zeta potential test was used to verify whether it was the electrostatic attraction drives silica and polydopamine together or not. Silica in solution is negatively charged and the potential is approx. $-17.3 \mathrm{mV}$, while polydopamine is also negatively charged and the potential is approx. $-2.77 \mathrm{mV}$. As these two are also negatively charged, it is hard to decide that the electrostatic attraction drives them together. However, we have found that there were some references suggested that hydrogen bonding between silicate species and chitin ${ }^{2,3}$ or hydroxylated collagen ${ }^{4}$ might be crucial in biosilica precipitation and morphologies. Meanwhile, there were also references reporting the utilization of polydopamine or L-DOPA to stabilize $\mathrm{Fe}_{3} \mathrm{O}_{4}$ in dispersions. ${ }^{5,6}$ The 
stabilization of $\mathrm{Fe}_{3} \mathrm{O}_{4}$ is attributed to the affinity anchor groups (hydroxyl groups). Similar to $\mathrm{Fe}_{3} \mathrm{O}_{4}$, there are also numerous hydroxyl groups at the superficial surface of the silica. Therefore, we regarded the hydrogen bonding as the strong interaction between the polydopamine and the surface of silica, which generates polydopamine bound HiFSNs clusters. 
The same samples used for TEM and FT-IR test were also employed for thermal gravimetric analysis (TGA) test. Initial dopamine concentration $(\mathrm{mg} / \mathrm{mL})$, weight loss (\%), and bound polydopamine (\%) were listed in Table S1.

Table S1. Weight loss for polydopamine bound HiFSNs clusters with different initial dopamine concentrations.

\begin{tabular}{ccc}
\hline $\begin{array}{c}\text { Initial dopamine concentration } \\
(\mathrm{mg} / \mathrm{mL})\end{array}$ & $\begin{array}{c}\text { Weight loss } \\
(\%)\end{array}$ & $\begin{array}{c}\text { Weight of Bound polydopamine }{ }^{\text {a) }} \\
(\%)\end{array}$ \\
\hline 0.00 & 0.349 & $/$ \\
0.05 & 0.520 & 0.171 \\
0.10 & 0.920 & 0.571 \\
0.50 & 2.800 & 2.447 \\
1.00 & 4.427 & 4.079 \\
\hline
\end{tabular}

a): The weight of the bound polydopamine was calculated by the formula,

$\Delta m_{\text {polydopamin } e}=\Delta m_{x}-\Delta m_{0}$

where the $\Delta m_{x}$ is the weight loss of the sample with $\mathrm{x} \mathrm{mg} / \mathrm{mL}$ initial dopamine concentration and $\Delta m_{0}$ is the weight loss of the sample with $0 \mathrm{mg} / \mathrm{mL}$ initial dopamine concentration. 
Microscale porous structure network was developed when abundant polydopamine bound HiFSNs clusters were excluded from adjacent ice crystals. The evolution of porous morphology at various temperatures was shown in Figure S5.

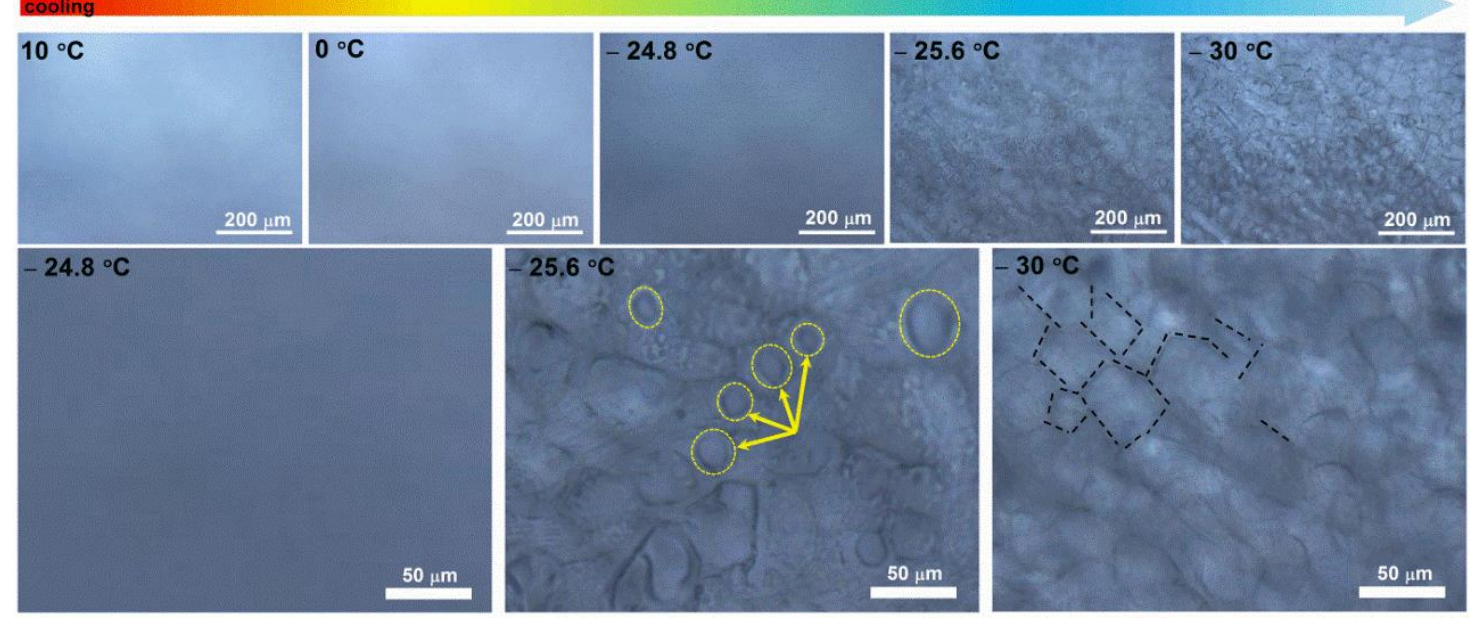

Figure S5. Top five images are PCM images taken during the cooling processing to the polydopamine bound HiFSNs cluster dispersion with initial dopamine concentration of $1.0 \mathrm{mg} / \mathrm{mL}$ and HiFSNs concentration of $1: 100$, wt/wt. Bottom three images are enlarged PCM images of the images taken at $-24.8^{\circ} \mathrm{C},-25.6{ }^{\circ} \mathrm{C}$, and $-30{ }^{\circ} \mathrm{C}$. During the cryogenic processing, the coated substrate was immediately placed at $-30{ }^{\circ} \mathrm{C}$. The temperature was dropped sharply. Therefore, we have selected two cooling rate, i.e., $5{ }^{\circ} \mathrm{C} / \mathrm{min}$, and $10{ }^{\circ} \mathrm{C} / \mathrm{min}$ to simulate the cryogenic processing. We found out that the coated dispersions were frozen at around $-25^{\circ} \mathrm{C}$ at either cooling rate. Hence, we chose the result obtained at the freezing rate of $10{ }^{\circ} \mathrm{C} / \mathrm{min}$ to demonstrate the ice formation in the dispersion. Yellow circles indicates the formation of ice bulks. Black dashed lines indicates the microscale porous structure network. 


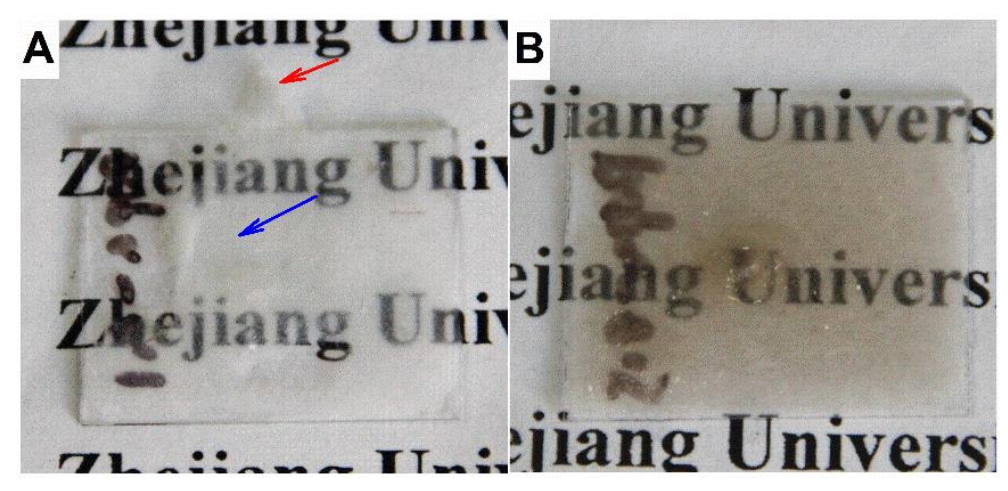

Figure S6. Photographs of coatings after freeze-drying process. (A) The coating was prepared by using dispersion with initial dopamine concentration of $0.1 \mathrm{mg} / \mathrm{mL}$. Blue arrow indicates the area of the glass slide that was not covered by the coating. Red arrow indicates the broken part of the coating. (B) The coating was prepared by using dispersion with initial dopamine concentration of $0.5 \mathrm{mg} / \mathrm{mL}$. 


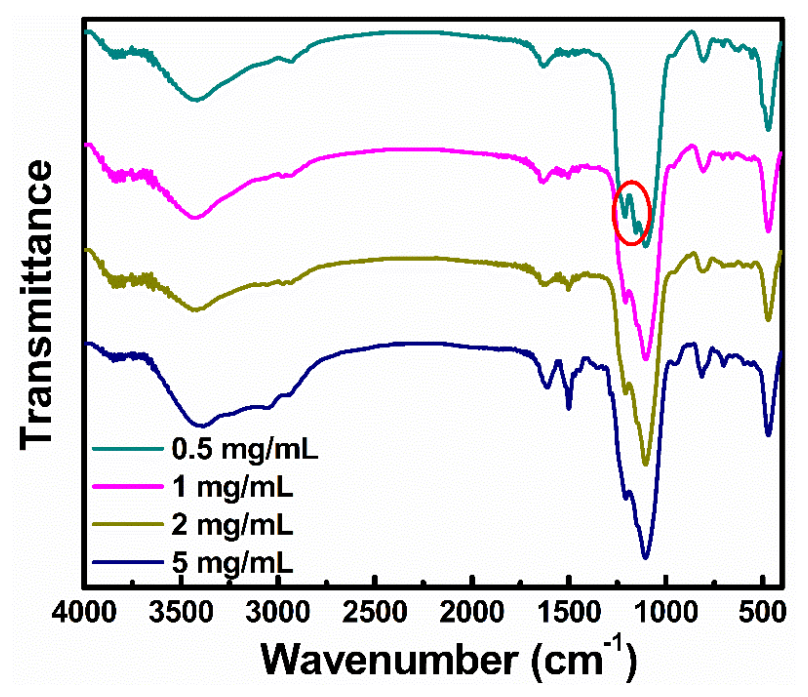

Figure S7. FT-IR spectra of the polydopamine bound HiFSNs clusters (i.e., porous coating) after the porous structure was treated with the two-step CVD. After CVD treatment, the polydopamine bound HiFSNs clusters were scraped from the coatings and undergone the FT-IR test. Red circle indicates the existence of $\mathrm{CF}_{2}$. 

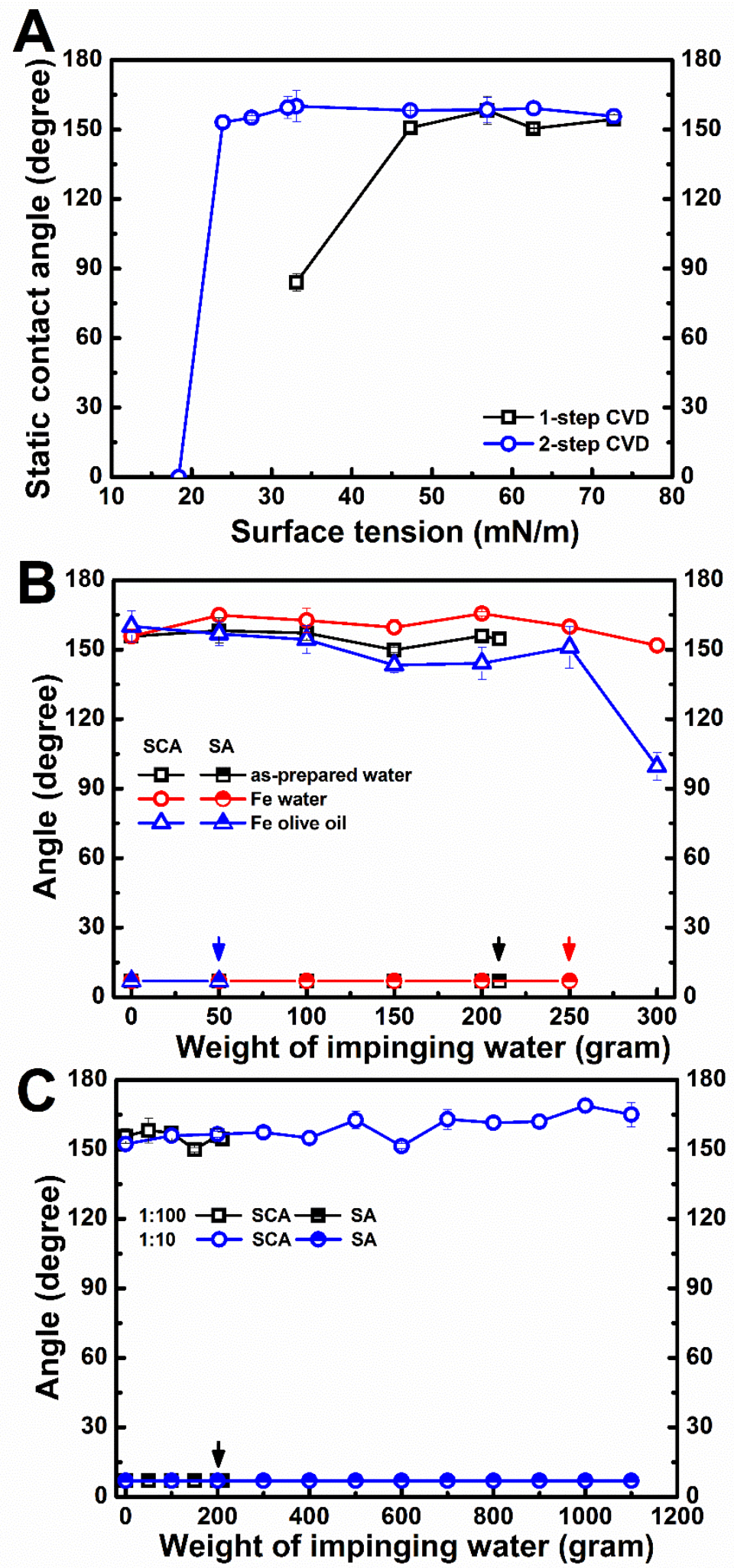

Figure S8. SCAs and SAs for coatings treated with different CVD processes and impinged by water drops. (A) SCAs for microscale porous coatings treated with onestep CVD $\left(1 \mathrm{H}, 1 \mathrm{H}, 2 \mathrm{H}, 2 \mathrm{H}-\right.$ perfluorodecyltriethoxysilane) and two-step CVD (1 $1^{\text {st. }} 3$ - 
aminopropyltriethoxysilane, $2^{\text {nd. }} \quad 1 \mathrm{H}, 1 \mathrm{H}, 2 \mathrm{H}, 2 \mathrm{H}$-perfluorodecyltriethoxysilane).

SCAs and SAs for water and olive oil droplets on the superamphiphobic coatings which were successively impinged by water droplets. "Fe" means that the coating was prepared by the dispersion which was added $12 \mu \mathrm{L} 1 \mathrm{M} \mathrm{FeCl}_{3}$. Arrows indicate the adherence of droplets on the tested coatings. (C) SCAs and SAs for coatings prepared with 1:10 wt/wt HiFSNs and 1:100 wt/wt HiFSNs. The initial dopamine concentrations are kept as $1 \mathrm{mg} / \mathrm{mL}$.
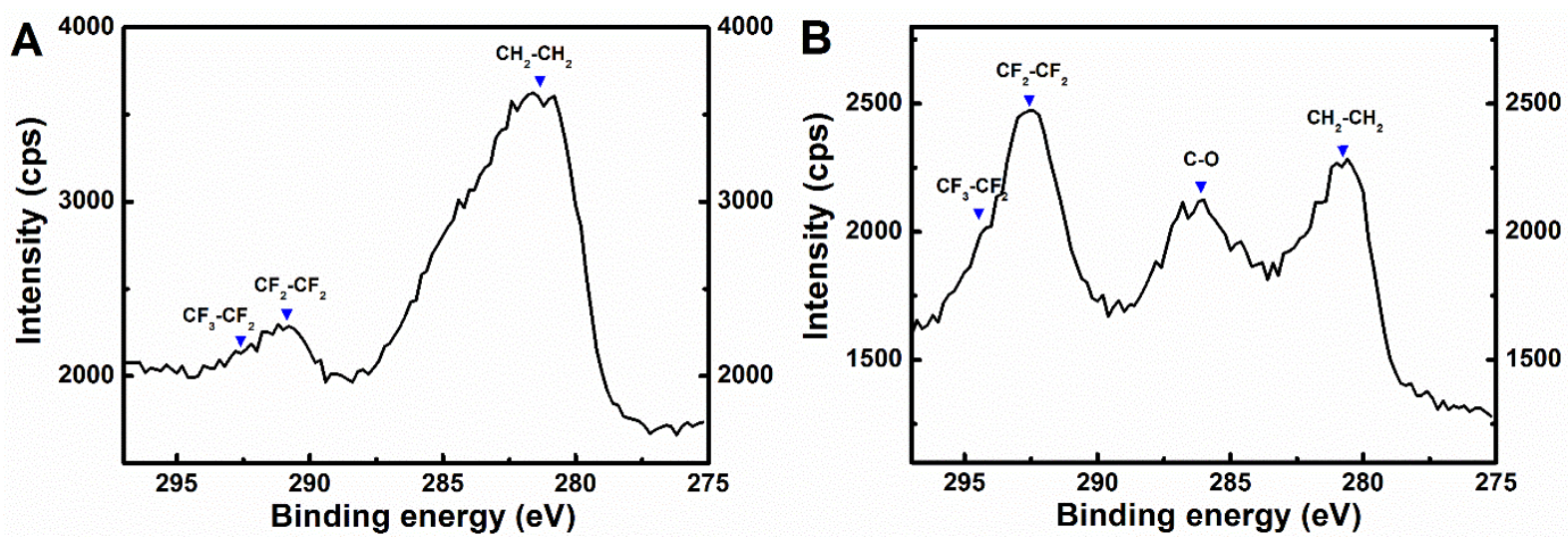

Figure S9. High resolution XPS spectra for C 1 s of the microscale porous coatings after (A) one-step CVD and (B) two-step CVD.

We have also prepared the coating which was directly CVD of $1 \mathrm{H}, 1 \mathrm{H}, 2 \mathrm{H}, 2 \mathrm{H}-$ perfluorodecyltriethoxysilane. SCAs dramatically decreased when olive oil (33.1 $\mathrm{mN} / \mathrm{m})$ droplet was deposited. And $\mathrm{CF}_{3}-\mathrm{CF}_{2}$ and $\mathrm{CF}_{2}-\mathrm{CF}_{2}(290 \mathrm{eV}-295 \mathrm{eV})$ occupied more outer surface than $\mathrm{CH}_{2}-\mathrm{CH}_{2}$ (approx. $281 \mathrm{eV}$ ) for the coating after two-step CVD. ${ }^{7,8} \mathrm{C}-\mathrm{O}$ peak can be attributed to the incompletely reacted $\mathrm{Si}-\mathrm{O}-\mathrm{C}_{2} \mathrm{H}_{5}$. It means that coating after has an outer layer with much lower surface energy. This can also 
explain the coating after two-step CVD could show better super-repellency than the coating after one-step CVD.

After impinged by $210 \mathrm{~g}$ of water, the as-prepared coating remained SCAs beyond $150^{\circ}$ for water (Figure S8B). Further impinging makes the coating lose superamphiphobicity. $\mathrm{Fe}^{3+}$ was reported can strengthen the robustness of the polydopamine coated surfaces by $\mathrm{Fe}^{3+}$ coordination. ${ }^{6,9,10} 12 \mu \mathrm{L} 1 \mathrm{M} \mathrm{FeCl}_{3}$ solution was added into the polydopamine bound HiFSNs dispersion before coating onto the substrate. The superamphiphobicity can last longer than the coating without $\mathrm{Fe}^{3+}$, but the robustness is still not good enough. The bottom layer of the coating could last longer than the whole coating, indicating that the adhesion strength is larger than the robustness of the coating. Increasing the concentration of HiFSNs makes the coating stand approx. $1100 \mathrm{~g}$ of water impinging (Figure S8C). However, in this case, the porous coating could only repel glycerol $\left(\gamma_{\text {glycerol }}=62.7 \mathrm{mN} / \mathrm{m}, \mathrm{SCAs}>157.5^{\circ}\right)$ and be completely wetted by olive oil. 


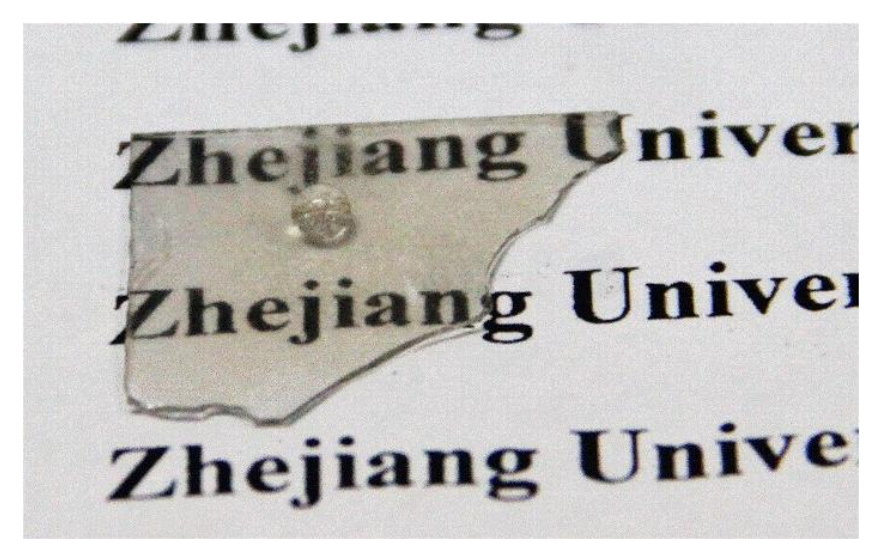

Figure S10. Photograph of a water droplet deposited on the quasi-superamphiphobic coating. Readable words under the coated surface indicated the transparency of the coating. 


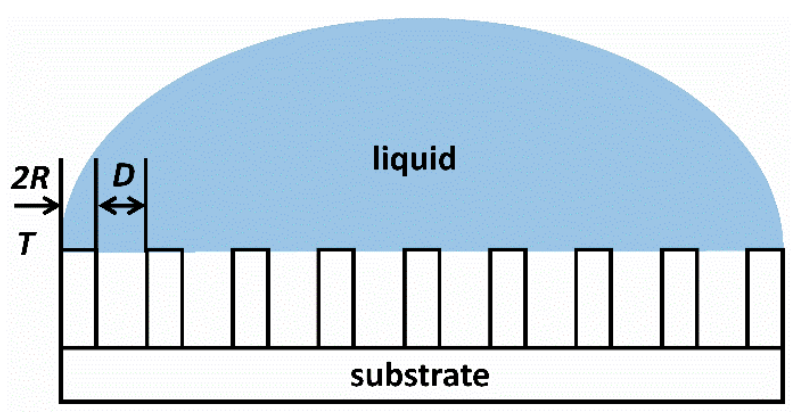

Figure S11. Schematic illustration for a droplet deposited on the porous structure. Assume that there are $n$ pores under the deposited droplet.

A

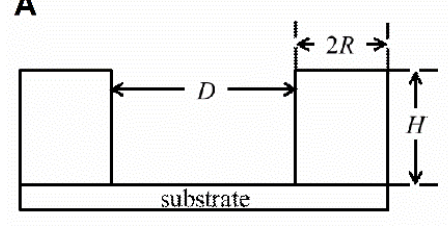

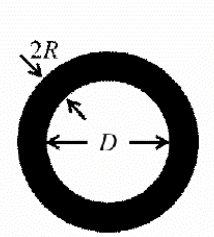

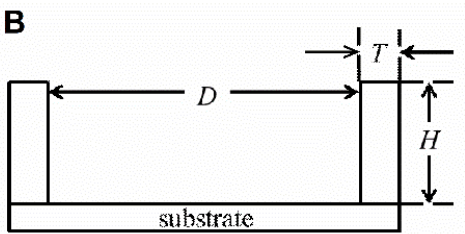

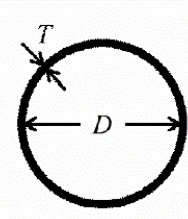

Figure S12. Schematic illustration for calculating the air fraction $\left(\phi_{\text {air }}\right)$ of the (A) nanoscale porous structure, and (B) microscale porous structure.

When a droplet is deposited on a porous structure (nanoscale or microscale), there are some pores under the droplet (Figure S11). Hypothesizing that there are $n$ pores under the deposited droplet, so there should be $n+1$ porous structure unit. For microscale porous structure (Figure S12B), the equation is written as,

$\phi_{\text {air }}=\frac{n D}{(n+1) T+n D}$.

As $D\left(D_{1}\right.$ and $\left.D_{2}\right)$ is significantly larger than $T\left(T_{1}\right.$ and $\left.T_{2}\right)$, Equation (S1a) could be approximately written as,

$\phi_{\text {air }}=\frac{n D}{n T+n D}$

and then Equation (S1b) can be simplified as,

$\phi_{\text {air }}=\frac{D}{T+D}$

Therefore, we got Equation (3b) and (3c). 
For nanoscale porous structure (Figure S12A), the equation is written as,

$$
\phi_{\text {air }}=\frac{n D}{(n+1) 2 R+n D} \text {. }
$$

The right side of the equation can be divided by $n$ both in numerator and denominator, and Equation (S2a) can be re-written as follows,

$$
\phi_{\text {air }}=\frac{D}{\frac{n+1}{n} 2 R+D} \text {. }
$$

When a droplet is deposited on a nanoscale porous structure, there are numerus pores under the droplet. $n$ possesses a very big value, making the value of $\frac{n+1}{n}$ close to 1 .

Therefore, Equation (2b) can be approximately given as,

$$
\phi_{\text {air }}=\frac{D}{2 R+D}
$$

Then, we get Equation (3a). 

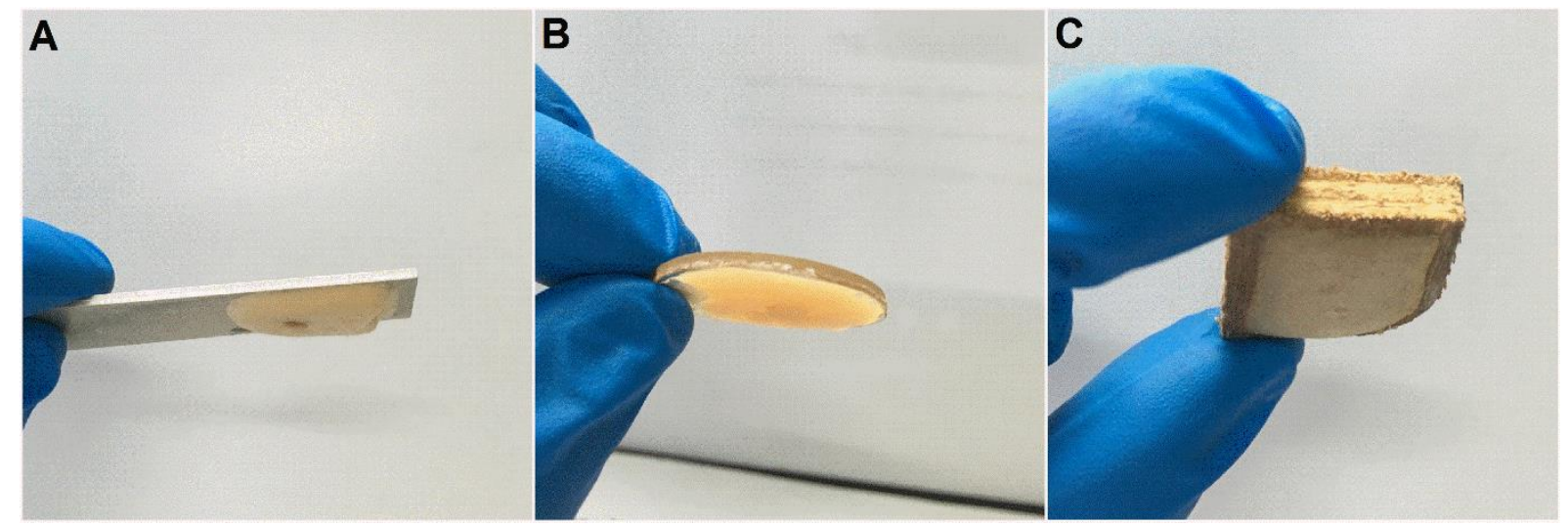

Figure S13. Microscale porous superamphiphobic coatings on (A) aluminum, (B)

PMMA, and (C) wood. The substrates were turned over to certify that the coatings were actually adhered.

\section{REFERENCES}

1. Lee, H.; Dellatore, S. M.; Miller, W. M.; Messersmith, P. B. Mussel-Inspired Surface Chemistry for Multifunctional Coatings. Science 2007, 318, 426-430.

2. Brunner, E.; Richthammer, P.; Ehrlich, H.; Paasch, S.; Simon, P.; Ueberlein, S.; van Pée, K.-H. ChitinBased Organic Networks: An Integral Part of Cell Wall Biosilica in the Diatom Thalassiosira pseudonana. Angew. Chem., Int. Ed. 2009, 48, 9724-9727.

3. Spinde, K.; Kammer, M.; Freyer, K.; Ehrlich, H.; Vournakis, J. N.; Brunner, E. Biomimetic Silicification of Fibrous Chitin from Diatoms. Chem. Mater. 2011, 23, 2973-2978.

4. Ehrlich, H.; Deutzmann, R.; Brunner, E.; Cappellini, E.; Koon, H.; Solazzo, C.; Yang, Y.; Ashford, D.; Thomas-Oates, J.; Lubeck, M.; Baessmann, C.; Langrock, T.; Hoffmann, R.; Wörheide, G.; Reitner, J.; Simon, P.; Tsurkan, M.; Ereskovsky, A. V.; Kurek, D.; Bazhenov, V. V.; Hunoldt, S.; Mertig, M.; Vyalikh, D. V.; Molodtsov, S. L.; Kummer, K.; Worch, H.; Smetacek, V.; Collins, M. J. Mineralization of the Metre-Long Biosilica Structures of Glass Sponges Is Templated on Hydroxylated Collagen. Nat Chem 2010, 2, 1084-1088.

5. Amstad, E.; Gillich, T.; Bilecka, I.; Textor, M.; Reimhult, E. Ultrastable Iron Oxide Nanoparticle Colloidal Suspensions Using Dispersants with Catechol-Derived Anchor Groups. Nano Lett. 2009, 9, 4042-4048.

6. Kim, S.; Gim, T.; Kang, S. M. Stability-Enhanced Polydopamine Coatings on Solid Substrates by Iron(III) Coordination. Prog. Org. Coat. 2014, 77, 1336-1339.

7. Chen, X. H.; Yang, G. B.; Kong, L. H.; Dong, D.; Yu, L. G.; Chen, J. M.; Zhang, P. Y. Direct Growth of Hydroxy Cupric Phosphate Heptahydrate Monocrystal with Honeycomb-Like Porous Structures on Copper Surface Mimicking Lotus Leaf. Crystal Growth \& Design 2009, 9, 2656-2661.

8. Cao, M.; Song, X.; Zhai, J.; Wang, J.; Wang, Y. Fabrication of Highly Antireflective Silicon Surfaces with Superhydrophobicity. J. Phys. Chem. B 2006, 110, 13072-13075.

9. Harrington, M. J.; Masic, A.; Holten-Andersen, N.; Waite, J. H.; Fratzl, P. Iron-Clad Fibers: A Metal- 
Based Biological Strategy for Hard Flexible Coatings. Science 2010, 328, 216-220.

10. Wilker, J. J. The Iron-Fortified Adhesive System of Marine Mussels. Angew. Chem., Int. Ed. 2010, 49, 8076-8078. 